\title{
Enhanced performance in polymer photovoltaic cells with chloroform treated indium tin oxide anode modification
}

\author{
Z. Q. Xu, ${ }^{1}$ J. Li, ${ }^{1}$ J. P. Yang, ${ }^{1}$ P. P. Cheng, ${ }^{1}$ J. Zhao, ${ }^{1}$ S. T. Lee, ${ }^{2}$ Y. Q. Li, ${ }^{1, a)}$ and \\ J. X. Tang ${ }^{1, a)}$ \\ ${ }^{1}$ Jiangsu Key Laboratory for Carbon-Based Functional Materials and Devices, Institute of Functional Nano \\ \& Soft Materials (FUNSOM), Soochow University, Suzhou 215123, People's Republic of China \\ ${ }^{2}$ Department of Physics and Materials Science, Center Of Super-Diamond and Advanced Films (COSDAF), \\ City University of Hong Kong, Hong Kong
}

(Received 20 April 2011; accepted 31 May 2011; published online 20 June 2011)

\begin{abstract}
Enhanced performance of a poly(3-hexylthiophene):(6,6)-phenyl $\mathrm{C}_{61}$ butyric acid methyl ester bulk heterojunction polymer photovoltaic cell is reported by modifying the indium tin oxide (ITO) anode with chloroform solution. Instead of the traditional UV-ozone treatment, the optimized chloroform modification on ITO anode can result in an enhancement in the power conversion efficiency of an identical device, originating from an increase in the photocurrent with negligible change in the open-circuit voltage. The performance enhancement is attributed to the work function modification of the ITO substrate through the surface incorporation of the chlorine, and thus improved charge collection efficiency. (C) 2011 American Institute of Physics. [doi:10.1063/1.3601853]
\end{abstract}

In recent years, polymer photovoltaic $(\mathrm{PV})$ cells are attracting tremendous interest, and the power conversion efficiency (PCE) has been reported to reach 6\%-7\%., ${ }^{1,2}$ Besides significant efforts on the bulk heterojunction (BHJ) photoactive layer, it has also been well recognized that the properties of interfaces between the photoactive layer and the electrode or an underlying buffer layer, e.g., Ohmic contacts, interfacial cohesion, or charge traps, are of fundamental importance to governing the PV performance because they can strongly impact the charge transport and collection process as well as the built-in potential. ${ }^{3-6}$ Therefore, control over these interfaces is essential to the PCE improvement in polymer PV cells. $^{7-9}$

Previous studies have been demonstrated that surface engineering to the indium tin oxide (ITO) anode with plasma treatment, ${ }^{10}$ UV-ozone treatment, ${ }^{11}$ self-assembled monolayers, ${ }^{5,12-14}$ or an oxide buffer layer, ${ }^{15}$ can increase the PCE for polymer PV cells by improving the charge collection efficiency with enhanced short-circuit current density $\left(\mathrm{J}_{\mathrm{SC}}\right)$. Among various methods to modify the ITO anode, oxygen plasma or UV-ozone treatment is the most widely used for the performance improvement of a polymer PV cell, which can remove the contamination as well as incorporate more oxygen onto the ITO surface for the work function $(\Phi)$ increase ${ }^{16}$ However, the spin casting of the aqueous solution of poly(3,4-ethylene dioxythiophene):polystyrene sulfonic acid (PEDOT:PSS), the widely used anode buffer layer in the polymer PV cells, inevitably reduces the efficiency of the oxidative treatments with UV-ozone. Consequently, it is necessary to find out a simple and efficiency approach to modify the ITO anode, which is compatible with the aqueous fabrication process of the PEDOT:PSS layer. Recently, Helander et $a l .{ }^{17}$ demonstrated a new method to enhance ITO surface work function by using o-dichlorobenzene and UV radiation, and significantly enhanced the device performance. In this letter, we report on an enhanced performance of polymer PV

\footnotetext{
a) Authors to whom correspondence should be addressed. Electronic addresses: jxtang@suda.edu.cn and yqli@suda.edu.cn.
}

cells by modifying the ITO anode with chloroform solution to replace the traditional UV-ozone treatment. An increase in $\mathrm{J}_{\mathrm{SC}}$ with negligible change in the open-circuit voltage $\left(\mathrm{V}_{\mathrm{OC}}\right)$ is realized for chloroform-treated polymer PV cells, resulting in a $15 \%$ overall efficiency improvement over the UV-ozone treated device.

The ITO-coated glass substrate with a sheet resistance of $20 \Omega /$ sq. was first cleaned following a routine solvent process. Then, the cleaned ITO glass substrates were treated by dipping into the chloroform solution for different time, and dried with $\mathrm{N}_{2}$ flow. The substrates were next spin-coated with a 40-nm-thick PEDOT:PSS layer, which was annealed in air at $140{ }^{\circ} \mathrm{C}$ for $30 \mathrm{~min}$. Photoactive layer, consisting of a blend of poly(3-hexylthiophene):(6,6)-phenyl $\mathrm{C}_{61}$ butyric acid methyl ester (P3HT:PCBM) with a 1:0.8 weight ratio and a thickness of $\sim 120 \mathrm{~nm}$ was formed on PEDOT:PSS coated ITO substrates in the nitrogen-filled glove box, and heated at $120{ }^{\circ} \mathrm{C}$ for $10 \mathrm{~min}$. After spin-coating the photoactive layer, the substrates were transferred to a high vacuum chamber (Mini-Spectros ${ }^{\circledR}$, Kurt J. Lesker) with a base pressure of $1 \times 10^{-6}$ Torr for thermal evaporation of a $\operatorname{LiF}(0.5$ $\mathrm{nm}) / \mathrm{Al}(100 \mathrm{~nm})$ bilayer cathode. A shadow mask was used to define the Al cathode, and the active device area determined by the overlap of ITO anode and Al cathode was estimated to be $0.1 \mathrm{~cm}^{2}$. Prior to subsequent measurements, the completed devices were postannealed through a digitally controlled hot plate at $150{ }^{\circ} \mathrm{C}$ for $30 \mathrm{~min}$ in the glove box. For comparison, the reference devices with as-cleaned and 15 min UV-ozone treated ITO anodes were also fabricated with the identical process parameters during the same batch processing with the chloroform-treated ones.

PV measurements were conducted at room temperature in air without any device encapsulation under the illumination of a $150 \mathrm{~W}$ Newport 91160 solar simulator using an air mass (AM) $1.5 \mathrm{G}$ filter. The simulated light intensity was adjusted to be $100 \mathrm{~mW} / \mathrm{cm}^{2}$, which was calibrated with a $\mathrm{Si}$ optical power meter. The current density-voltage (J-V) characteristics were examined with a programmable Keithley 2612 source meter. The incident photon to current conver- 


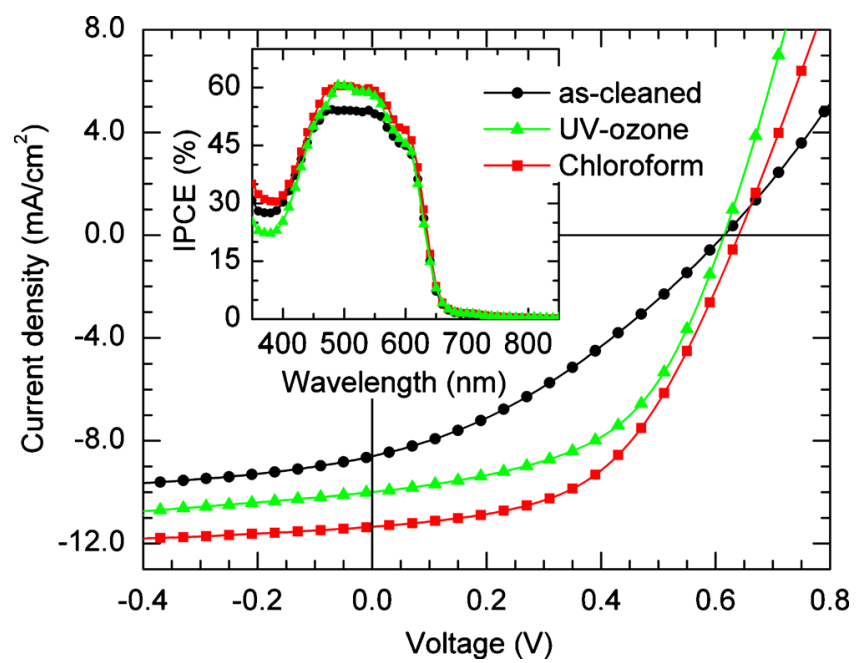

FIG. 1. (Color online) J-V characteristics of polymer PV cells with a structure of ITO/PEDOT:PSS/P3HT:PCBM/LiF/Al under $100 \mathrm{~mW} / \mathrm{cm}^{2} \mathrm{AM}$ $1.5 \mathrm{G}$ simulated solar illuminations. Inset is the corresponding IPCE spectra.

sion efficiency (IPCE) spectra were measured with a photomodulation spectroscopic setup (Newport monochromator).

Figure 1 shows the $\mathrm{J}-\mathrm{V}$ characteristics of the polymer PVs with different treated ITO anodes under AM 1.5G simulated solar illumination at an incident intensity of $100 \mathrm{~mW} / \mathrm{cm}^{2}$. The extracted device parameters are summarized in Table I. As evident in Fig. 1, the $\mathrm{V}_{\mathrm{OC}}$ remains almost unchanged for devices with different ITO treatments, while the $\mathrm{J}_{\mathrm{SC}}$ and fill factor (FF) are significantly enhanced. Compared to devices with as-cleaned and UV-ozone treated ITO anodes, the PV cell with chloroform treatment for $40 \mathrm{~min}$ shows an enhanced performance with a PCE of $3.68 \%$, a $\mathrm{FF}$ of $50.7 \%$, a $\mathrm{V}_{\mathrm{OC}}$ of $0.64 \mathrm{~V}$, and a $\mathrm{J}_{\mathrm{SC}}$ of $11.37 \mathrm{~mA} / \mathrm{cm}^{2}$, respectively.

To get additional insight into the device performance, the IPCE or external quantum efficiency of the corresponding PV cells are compared (see the inset of Fig. 1). For the entire wavelength range, the PV cell with chloroform-treated ITO anode exhibits a higher IPCE than the other two cases. In addition, there is no noticeable difference in shape of the IPCE spectra among these PV cells, implying that the morphology and crystallinity degree of the P3HT:PCBM BHJ in each cell are similar and have no significant influence on the charge generation and transport. Therefore, the performance enhancement with chloroform treatment cannot be attributed to the photoactive layers.

To understand the origin of the observed $\mathrm{J}_{\mathrm{SC}}$ and PCE enhancements with respect to the surface treatment, optical transmission spectra of the ITO glass substrates with different treatments were measured with a Perkin Elmer Lambda

TABLE I. Device performance parameters of polymer PV cells with different treated ITO anodes.

\begin{tabular}{lcccccc}
\hline \hline \multicolumn{1}{c}{ ITO } & $\begin{array}{c}\Phi \\
(\mathrm{eV})\end{array}$ & $\begin{array}{c}\mathrm{V}_{\mathrm{OC}} \\
(\mathrm{V})\end{array}$ & $\begin{array}{c}\mathrm{J}_{\mathrm{SC}} \\
\left(\mathrm{mA} / \mathrm{cm}^{2}\right)\end{array}$ & $\begin{array}{c}\mathrm{FF} \\
(\%)\end{array}$ & $\begin{array}{c}\text { PCE } \\
(\%)\end{array}$ & $\begin{array}{c}\mathrm{R}_{\mathrm{S}} \\
(\Omega)\end{array}$ \\
\hline As-cleaned & 4.58 & 0.61 & 8.60 & 34.3 & 1.80 & 42.0 \\
UV-Ozone & 4.75 & 0.61 & 9.99 & 52.2 & 3.18 & 15.5 \\
Chloroform 20 min & 4.70 & 0.62 & 11.49 & 45.4 & 3.23 & 23.3 \\
Chloroform 40 min & 4.80 & 0.64 & 11.35 & 50.7 & 3.68 & 18.3 \\
Chloroform 60 min & 4.86 & 0.64 & 9.67 & 46.8 & 2.85 & 28.7 \\
\hline \hline
\end{tabular}

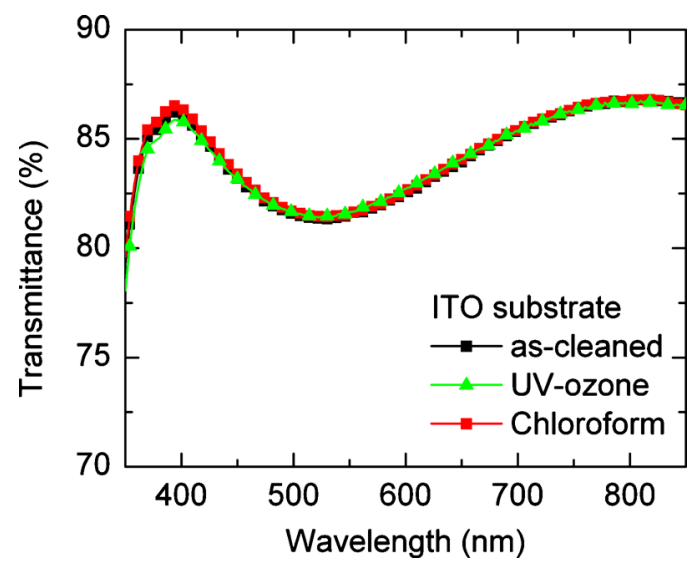

FIG. 2. (Color online) Optical transmittance spectra of the ITO substrates with different surface modification.

2S UV/visible spectrometer. As shown in Fig. 2, the optical transmittance remains almost identical to each other at the visible range, indicating no measurably influence of the surface modification on the transmittance and no incident light loss delivered to the active region under illumination.

The influence of various treatments on the $\Phi$ of ITO substrates was also investigated by ultraviolet and X-ray photoemission spectroscopy (UPS and XPS) in a Kratos AXIS Ultra-DLD ultrahigh vacuum photoemission spectroscopy system with HeI excitation $(\mathrm{h} \nu=21.22 \mathrm{eV})$ and monochromatic $\mathrm{Al} K \alpha$ source $(1486.6 \mathrm{eV})$. Details of the experimental setup for UPS and XPS measurements have been given elsewhere. ${ }^{18}$ Fig. 3 shows the secondary electron cutoff of UPS spectra for the corresponding ITO anodes with and without a 40-nm-thick PEDOT:PSS layer. As evident in Fig. 3 , the secondary electron cutoffs shift toward lower binding energy, indicating the increase in $\Phi$ with different treatments. In particular, the chloroform treatment can significantly increase the ITO's $\Phi$ as compared to that of as-cleaned and UV-ozone treated ITO (as summarized in Table I). As determined by the XPS analysis (see Fig. 4), the intensity of the Cl $2 p$ core level peak increases with chloroform treatment time, indicating that the increase of $\Phi$ induced by chloroform treatment is associated with the surface incorporation of

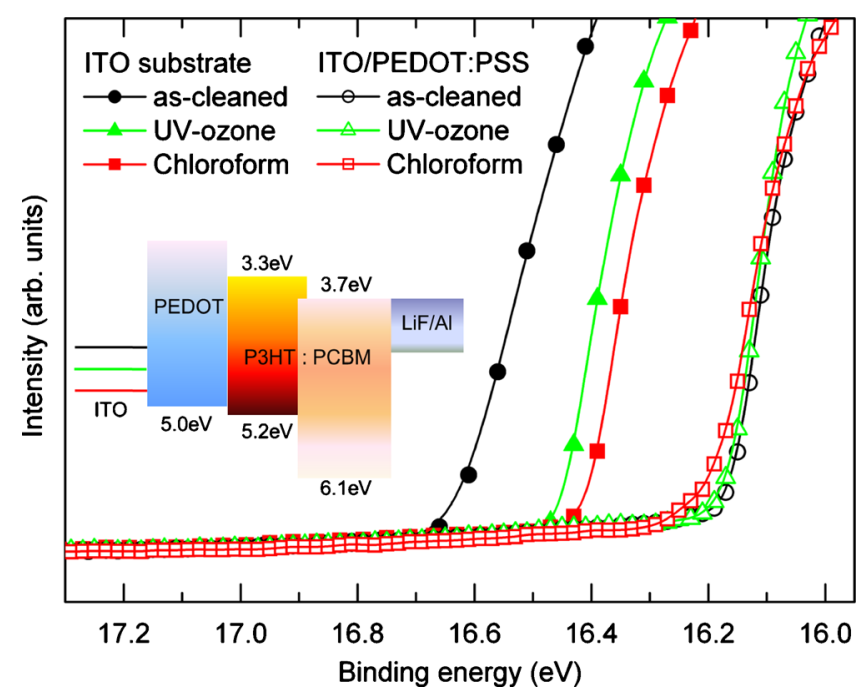

FIG. 3. (Color online) The secondary electron cutoff region of the UPS spectra of ITO anodes (closed symbols) and PEDOT:PSS (open symbols) on ITO substrates with different treatments. Inset is the schematic energy level diagram of the polymer PV cells with different ITO anodes. 


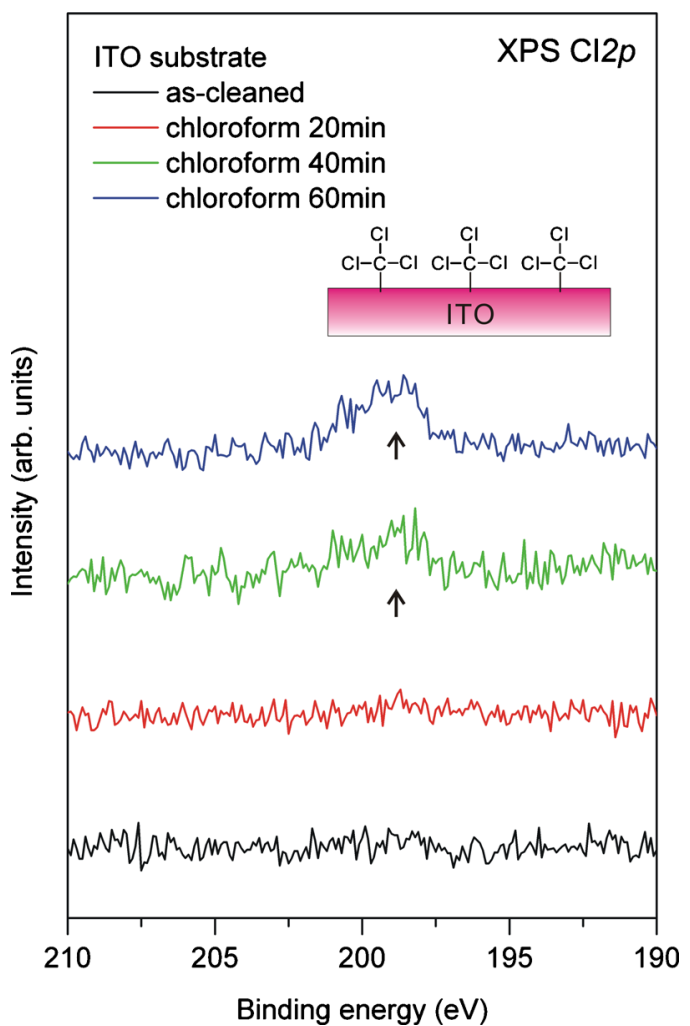

FIG. 4. (Color online) XPS Cl $2 p$ core level spectra of ITO substrates as a function of chloroform treatment time.

chlorine species and the resultant charged film surface. In contrast, as shown in Fig. 3, negligible changes in $\Phi$ of the PEDOT:PSS layer were induced by the spin casting of a PEDOT:PSS layer on different ITO anodes. It implies that the energy barrier at the interface between PEDOT:PSS and P3HT:PCBM will not be influenced by the treatment on ITO substrates. According to the proposed energy level diagram depicted in the inset of Fig. 3, the $\mathrm{J}_{\mathrm{SC}}$ enhancement for polymer PV cells with surface modification might be attributed to the creation of an interface energy step between ITO and PEDOT:PSS, leading to the reduced energy barrier and the improvement of charge collection efficiency and thus the PCE increase. ${ }^{12,19}$

A correlation between the time of chloroform treatment and the resulting device performance in PV cells was examined, and the characteristics of the chloroform-treated PV devices with different treatment time are summarized in Table I. Obviously, the $\Phi$ of chloroform treated ITO anodes increases with respect to the treatment time, which is accompanied with the increase in the $\mathrm{Cl} 2 p$ signals as observed in Fig. 4, indicating an increase in surface coverage of $\mathrm{Cl}$ species. However, in parallel with the increase in $\mathrm{J}_{\mathrm{SC}}$ and PCE, the chloroform treatment on the ITO anode exhibits a negligible effect on the $\mathrm{V}_{\mathrm{OC}}$. In particular, an optimized improvement on $\mathrm{J}_{\mathrm{SC}}$ and $\mathrm{FF}$ is realized for polymer PV cells with chloroform treatment of $40 \mathrm{~min}$, although that of $60 \mathrm{~min}$ causes the largest $\Phi$. This can be understood that the $\mathrm{V}_{\mathrm{OC}}$ is somehow determined by the energy difference of the highest occupied molecular orbital of the P3HT donor and the lowest unoccupied molecular orbital of the PCBM acceptor. On the other hand, the trend in $\mathrm{J}_{\mathrm{SC}}$ and $\mathrm{FF}$ with respect to different time of chloroform treatment can be related to the contact resistance of the chloroform-treated ITO anode. As shown in Table I, the series resistance $\left(R_{S}\right)$ of the ITO anode with a 40 min chloroform treatment is lower than those of $20 \mathrm{~min}$ and 60 min, respectively. As a result, a higher $\mathrm{R}_{\mathrm{S}}$ can lead to a reduction in $\mathrm{FF}$ and $\mathrm{J}_{\mathrm{SC}}$, hence a reduction in PCE, although the $\mathrm{V}_{\mathrm{OC}}$ is not significantly affected. ${ }^{20}$

In conclusion, we have demonstrated a simple and efficient method to modify the ITO anode for the performance enhancement in polymer PV cells. The $\mathrm{J}_{\mathrm{SC}}$ of the chloroform-treated device is increased with negligible change in the $\mathrm{V}_{\mathrm{OC}}$, as compared to the devices based on as-cleaned and UV-ozone treated ITO anodes. Under the optimized chloroform treatment, the PCE of chloroform-treated PV cells is $15 \%$ increased over UV-ozone treated device. The improved overall efficiency in PV devices is associated with the improved charge collection efficiency, due to the increase in ITO work function and thereby the better energy level alignment between ITO anode and the PEDOT:PSS buffer layer. These results demonstrate that the optimized chloroform treatment reported here defines a promising pathway for further improving the performance of polymer solar cells.

The authors acknowledge the financial support by the National Natural Science Foundation of China (Grant Nos. 91027041, 61007020, 61036009, and 60937001), 973 Program (Grant Nos. 2011CB808404 and 2010CB934502), 863 Program (Grant No. 2008AA03A327), the Research Fund for the Doctoral Program of Higher Education of China (Grant Nos. 20093201120019 and 20103201120019), and A Project Funded by the Priority Academic Program Development of Jiangsu Higher Education Institutions (PAPD).

${ }^{1}$ S. H. Park, A. Roy, S. Beaupre, S. Cho, N. Coates, J. S. Moon, D. Moses, M. Leclerc, K. Lee, and A. J. Heeger, Nat. Photonics 3, 297 (2009).

${ }^{2}$ H.-Y. Chen, J. Hou, S. Zhang, Y. Liang, G. Yang, Y. Yang, L. Yu, Y. Wu, and G. Li, Nat. Photonics 3, 649 (2009).

${ }^{3}$ L.-M. Chen, Z. Xu, Z. R. Hong, and Y. Yang, J. Mater. Chem. 20, 2575 (2010).

${ }^{4}$ X. Bulliard, S. G. Ihn, S. Yun, Y. Kim, D. Choi, J.-Y. Choi, M. Kim, M. Sim, J.-H. Park, W. Choi, and K. Cho, Adv. Funct. Mater. 20, 4381 (2010).

${ }^{5}$ H. Ma, H.-L. Yip, F. Huang, and A. K.-Y. Jen, Adv. Funct. Mater. 20, $1371(2010)$

${ }^{6}$ B. C. Thompson and J. M. J. Fréchet, Angew. Chem., Int. Ed. 47, 58 (2007)

${ }^{7}$ K. Schulze, B. Maennig, K. Leo, Y. Tomita, C. May, J. Hüpkes, E. Brier, E. Reinold, and P. Bäuerle, Appl. Phys. Lett. 91, 073521 (2007).

${ }^{8}$ F. Yang and S. R. Forrest, Adv. Mater. (Weinheim, Ger.) 18, 2018 (2006).

${ }^{9}$ C. Ko, Y. Lin, F. Chen, and C. Chu, Appl. Phys. Lett. 90, 063509 (2007).

${ }^{10}$ Z. Hong, C. Liang, X. Sun, and X. Zeng, J. Appl. Phys. 100, 093711 (2006).

${ }^{11}$ P. Destruel, H. Bock, I. Seguy, P. Jolinat, M. Oukachmih, and E. BedelPereira, Polym. Int. 55, 601 (2006).

${ }^{12}$ S. Khodabakhsh, B. M. Sanderson, J. Nelson, and T. S. Jones, Adv. Funct. Mater. 16, 95 (2006).

${ }^{13}$ C. Goh, S. R. Scully, and M. D. McGehee, J. Appl. Phys. 101, 114503 (2007)

${ }^{14}$ T. C. Monson, M. T. Lloyd, D. C. Olson, Y.-J. Lee, and J. W. P. Hsu, Adv. Mater. (Weinheim, Ger.) 20, 4755 (2008).

${ }^{15}$ W.-J. Yoon and P. R. Berger, Appl. Phys. Lett. 92, 013306 (2008).

${ }^{16}$ M. G. Mason, L. S. Hung, C. W. Tang, S. T. Lee, K. W. Wong, and M. Wang, J. Appl. Phys. 86, 1688 (1999).

${ }^{17}$ M. G. Helander, Z. B. Wang, J. Qiu, M. T. Greiner, D. P. Puzzo, Z. W. Liu, and Z. H. Lu, Science 332, 944 (2011).

${ }^{18}$ H. X. Wei, J. Li, Z. Q. Xu, Y. Cai, J. X. Tang, and Y. Q. Li, Appl. Phys. Lett. 97, 083302 (2010).

${ }^{19}$ M. D. Irwin, J. Liu, B. J. Leever, J. D. Servaites, M. C. Hersam, M. F. Durstock, and T. J. Marks, Langmuir 26, 2584 (2010).

${ }^{20}$ J. G. Xue, S. Uchida, B. P. Rand, and S. R. Forrest, Appl. Phys. Lett. 84 3013 (2004). 